\title{
Specification testing under moment inequalities
}

\author{
Patrik Guggenberger $^{\mathrm{a}, *}$, Jinyong Hahn ${ }^{\mathrm{a}}$, Kyooil Kim ${ }^{\mathrm{b}}$ \\ a Department of Economics, UCLA, USA \\ ${ }^{\mathrm{b}}$ Department of Economics, University of Minnesota, USA
}

Received 3 April 2006; received in revised form 7 August 2007; accepted 4 September 2007

Available online 11 September 2007

\begin{abstract}
We provide a specification test for moment inequalities based on a dual characterization of the moment inequalities. For linear moment inequalities, the test is the asymptotic version of the multi-dimensional linear one-sided tests. For nonlinear moment inequalities, the implementation of the test is not practical because the dual characterization takes the form of a multi-dimensional nonlinear one-sided hypothesis. (C) 2007 Elsevier B.V. All rights reserved.
\end{abstract}

Keywords: Moment inequalities; Partial identification; Specification test

JEL classification: $\mathrm{C} 12$

\section{Introduction}

There has been a recent surge of interest in statistical inference in situations where parameters of interest are only partially identified. See Manski (2003) for an overview of this literature. In some applications, the parameter is real-valued and the identified set is an interval whose lower and upper bounds may be estimated from the sample. A confidence interval (CI) of the identified set may be constructed by taking account of the sampling variation of these estimates. The CI may be constructed to cover the entire identified or the true value of the parameter with a certain fixed probability. Imbens and Manski (2004), who proposed the latter, observed that the two CIs can be quite different. The difference of the widths of the two CIs can be related to the difference in critical values of onesided and two-sided tests.

The purpose of this note is to extend Imbens and Manski's (2004) insight to a situation where the parameter of interest is

\footnotetext{
* Corresponding author. Bunche Hall 8385, Department of Economics UCLA, Box 951477, Los Angeles, CA 90095-1477, USA. Tel.: +1 310825 0849; fax: +13108259528.

E-mail address: guggenbe@econ.ucla.edu (P. Guggenberger).
}

multi-dimensional and can be characterized by moment inequalities. ${ }^{1}$ We propose a specification test to test whether such moment inequalities can hold by providing a dual characterization of the moment inequalities. For a model characterized by linear moment inequalities, we find that such a test is the asymptotic version of the multi-dimensional linear one-sided tests as discussed by, e.g., Gourieroux et al. (1982). On the other hand, when the model is given by nonlinear moment inequalities, the test will be subject to practical problems of implementation because the dual characterization takes the form of multidimensional nonlinear one-sided hypothesis. Wolak (1991) noted that the main difficulties of the nonlinear one-sided hypothesis tests are (i) "the lack of an empirically implementable procedure for computing an asymptotically exact size critical value", (ii) "the absence of tight upper and lower bounds on the asymptotic distribution of the test statistics", and (iii) "the least favorable null asymptotic distribution may not occur at the unique parameter value satisfying all of the inequality constraints

\footnotetext{
${ }^{1}$ Models characterized by moment inequalities have been recently considered by Moon and Schorfheide (2004) and Pakes et al. (2005). Other related papers include Manski and Tamer (2002), Andrews et al. (2004), Andrews and Guggenberger (2005), Beresteanu and Molinari (2005), Rosen (2005), Romano and Shaikh (2006), and Chernozhukov et al. (2007).
} 
with equality". Our dual characterization of the models with nonlinear moment inequalities suggests that these problems in the nonlinear one-sided hypothesis tests will be carried over to our specification test. We suspect that specification tests of nonlinear models with inequalities will remain elusive unless there is progress in testing of nonlinear one-sided hypothesis.

\section{Dual characterization of moment inequalities}

We first consider a linear model given by a restriction of the form $C \theta \leq \mu$ for $C \in R^{m \times d}, \mu \in R^{m}$, and $\theta \in R^{d}{ }^{2}$. We are interested in the specification test

$H_{0}: \exists \theta$ such that $C \theta \leq \mu$.

Example. Consider the linear regression model

$y_{i}=x_{i} \theta+\varepsilon_{i}$ and $E\left[x_{i} \varepsilon_{i}\right]=0$

for $\theta \in R^{d}$. Assume the range space of $y_{i}$ is partitioned into a certain number of disjoint intervals. For example, income of an individual is often times reported as an interval rather than a specific value. We do not actually observe $y_{i}$ but instead only observe the lower and upper interval bounds, denoted by $y_{i \mathrm{~L}}$ and $y_{i \mathrm{U}}$ respectively, of the interval that $y_{i}$ is part of, $y_{i} \in\left[y_{i \mathrm{~L}}\right.$, $\left.y_{i \mathrm{U}}\right)$. Assume $x_{i}$ has bounded support. Then w.l.o.g. we can assume that $x_{i} \geq 0$. It follows that

$E\left[x_{i} y_{i \mathrm{~L}}\right] \leq E\left[x_{i} y_{i}\right] \leq E\left[x_{i} y_{i \mathrm{U}}\right]$.

Therefore, writing $A=E\left[x_{i} x_{i}^{\prime}\right], \mu^{\mathrm{L}}=E\left[x_{i} y_{i \mathrm{~L}}\right]$, and $\mu^{\mathrm{U}}=E\left[x_{i} y_{i \mathrm{U}}\right]$, we obtain the restriction, $\mu^{\mathrm{L}} \leq A \theta \leq \mu^{\mathrm{U}}$ or $C \theta \leq \mu$ for $C=$ $\left(A^{\prime},-A^{\prime}\right)^{\prime}$ and $\mu=\left(\mu^{\mathrm{U}_{\prime}^{\prime}},-\mu^{\mathrm{L}}\right)^{\prime}$. The specification test in Eq. (1) tests whether there exist a $\theta \in R^{d}$ such that Eq. (2) holds.

We show that the null hypothesis (1) can be given a dual characterization of the form

$H_{0}: B \mu \geq 0$.

More precisely, we show that there exists a matrix $B=B(C)$ such that there is a $\theta$ satisfying $C \theta \leq \mu$ if and only if $B \mu \geq 0$. See the Appendix for an algorithm that generates such a matrix $B$. Note that the hypothesis (3) is the multi-dimensional one-sided test discussed, e.g., by Gourieroux et al. (1982) and Wolak (1991). If $C$ is known, then $B$ is known, and the test can be based on the Wald-type test statistic of the form

$W_{n}=\inf _{t \in R^{p}}\left\{n(B \widehat{\mu}-t)^{\prime} \widehat{J}^{-1}(B \widehat{\mu}-t)\right.$ subject to $\left.t \geq 0\right\}$,

where $\widehat{\mu}$ is a $\sqrt{n}$-consistent asymptotically normal estimator of $\mu$ and $\widehat{J}$ is a consistent estimator for the asymptotic variance matrix of $B \widehat{\mu}^{3}{ }^{3}$ The asymptotic distribution of $W_{n}$ is a mixture of $\chi^{2}$-distributions, see Kudo (1963).

\footnotetext{
${ }^{2}$ If $x$ and $y$ are both $m$-vectors, $x \leq y$ means $x_{i} \leq y_{i}$ for $i=1, \ldots, m$.

${ }^{3}$ If $B$ is unknown but a $\sqrt{n}$-consistent asymptotically normal estimator $\hat{B}$ of $B$ is available, then $\widehat{J}$ in $W_{n}$ need to be replaced by a consistent estimator of the asymptotic covariance matrix of $\widehat{B} \widehat{\mu}$.
}

Assume now that the model is given by $E[\varphi(w ; \theta)] \geq \mu$ where $\varphi$ is a nonlinear function with values in $R^{m}$. Letting $\Phi(\theta) \equiv\left(\Phi_{1}(\theta), \ldots, \Phi_{m}(\theta)\right)^{\prime} \equiv E[\varphi(w ; \theta)]$,we can write the null hypothesis as

$H_{0}: \exists \theta$ such that $\Phi(\theta) \geq \mu$.

Assume $\theta \in \Theta \subset R^{d}$ for some set $\Theta$. To derive the dual version of Eq. (5) define functions $\bar{\Phi}_{j}, j=1, \ldots, m$ :

1. Let $\bar{\Phi}_{1} \equiv \max _{\theta \in \Theta} \Phi_{1}(\theta)$.

2. For $j \geq 2$, let $\bar{\Phi}_{j} \equiv \bar{\Phi}_{j}\left(\mu_{1}, \ldots, \mu_{j-1}\right) \equiv \max _{\theta \in \Theta_{j}} \Phi_{j}(\theta)$ if $\Theta_{j} \neq \varnothing$, where $\Theta_{j} \equiv\left\{\theta \in \Theta: \Phi_{j}{ }^{\prime}(\theta) \geq \mu_{j}{ }^{\prime}\right.$ for all $\left.j^{\prime}<j\right\}$.

Let $\bar{\Phi} \equiv\left(\bar{\Phi}_{1}, \ldots, \bar{\Phi}_{m}\right)^{\prime}$. Clearly, there exists a $\theta$ such that $\Phi(\theta) \geq \mu$ if and only if $\Psi(\mu) \equiv \bar{\Phi}(\mu)-\mu \geq 0$ and thus Eq. (5) holds if and only if

$\Psi(\mu) \geq 0$

If we have a $\sqrt{n}$-consistent, asymptotically normal estimator $\hat{\mu}$ of $\mu$, testing Eq. (5) is equivalent to testing the one-sided hypothesis on $\mu$. The latter has been studied in Wolak (1991) using a Wald-type statistic as in Eq. (4). In order to determine the asymptotic critical value, one has to maximize the probability of rejection over all $\mu$ vectors that satisfy the restriction (6). However, as discussed in Wolak (1991), such a maximization and therefore determination of a critical value typically is computationally intractable. ${ }^{5}$

\section{Discussion: CI for a scalar component of $\boldsymbol{\theta}$}

The discussion in the preceding section has natural implications for the construction of a CI for a scalar component of a vector-valued parameter. Suppose for simplicity that the model is given by a set of linear restrictions $C \theta \leq \mu$. We are interested in testing whether the first component $\theta_{1}$ of $\theta$ is equal to $\theta_{1}^{*}$. We can write the null hypothesis as

$H_{0}: \exists \theta_{-1}$ such that $C_{-1} \theta_{-1} \leq \mu-c_{1} \theta_{1}^{*}$,

where $c_{1}$ is the first column of $C, C_{-1}$ is a submatrix consisting of the remaining columns, and $\theta_{-1}=\left(\theta_{2}, \ldots, \theta_{d}\right)^{\prime}$. The CI for $\theta_{1}$ can in principle be obtained as a set of all $\theta_{1}^{*}$ which are not rejected by the test of the above hypothesis. The same intuition implies that the confidence interval of a scalar component can be very difficult to construct when a model is given by nonlinear moment restrictions.

\footnotetext{
${ }^{4}$ We assume the maximum exists. We also assume that $\bar{\Phi}_{j}$, thus defined, is differentiable on the set $\left\{\mu \in R^{m} ; \Theta_{j} \neq \varnothing\right\}$. On the set $\left\{\mu \in R^{m} ; \Theta_{j}=\varnothing\right\}$ we define $\bar{\Phi}_{j}(\mu)$ such that the extended function $\bar{\Phi}_{j}$ is differentiable on $R^{m}$.

${ }^{5}$ Wolak's (1991) Lemma 1 (3) establishes that the maximum probability of rejection is achieved in some particular set $\mathbb{B} \equiv C^{b}-\left\{\mu: \mu \in C^{b}\right.$ and $\Psi_{j}(\mu)=$ 0 for only one $j=1, \ldots, m\}$, where $C^{b} \equiv\left\{\mu: \Psi_{j}(\mu) \geq 0, j=1, \ldots, m\right\}-\left\{\mu: \Psi_{j}\right.$ $(\mu)>0, j=1, \ldots, m\}$. The nonlinearity implies that we cannot further reduce the set of potential maximizers. This implies that in practice we need to simulate the distribution of Wolak's (1991) test statistic over $\mathbb{B}$, which is generally an impossible computational task.
} 
We note that the confidence region of the entire parameter $\theta$ is straightforward to implement from this perspective. In order to understand this point, continue to suppose that the model is given by $C \theta \leq \mu$. If we want to test whether $\theta=\theta_{*}$, all we need to do is to test whether $H_{0}: C \theta_{*} \leq \mu$. Such a test does not even require any dual characterization. By applying the asymptotic version of Gourieroux et al. (1982) test and comparing $C \theta_{*}$ with $\widehat{\mu}$, the confidence region for $\theta_{*}$ can be trivially obtained. In fact, a confidence region for $\theta$ can be constructed even for models characterized by nonlinear restrictions $E\left[\varphi\left(w_{i} ; \theta\right)\right] \leq \mu$ as above. If we are interested in testing $\theta=\theta_{*}$, it can be done again by comparing the sample analog $n^{-1} \sum_{i=1}^{n} \varphi\left(w_{i} ; \theta_{*}\right)$ of $E\left[\varphi\left(w_{i} ; \theta_{*}\right)\right]$ with $\widehat{\mu}$. Again, this can be done by applying the asymptotic version of Gourieroux et al.'s (1982) test. Although such a confidence region is rather straightforward to construct, at least conceptually, this does not imply that the CI for a scalar component is as easy to construct. See, e.g., Savin (1984), for related discussion.

\section{Acknowledgment}

We would like to thank G,nter M. Ziegler for extremely helpful correspondence on the topic of this paper. We would also like to thank the editor and an anonymous referee for helpful suggestions.

\section{Appendix A. Dual Characterization in Linear Models}

The goal is provide an easily implementable algorithm that yields a matrix $B=B(C)$ with minimal number of rows such that the null hypothesis (1) $\exists \theta$ such that $\left(C,-I_{m}\right)\left(\theta^{\prime}, \mu^{\prime}\right)^{\prime} \leq 0$ holds if and only if Eq. (3) holds. Algorithm 1 constructs a matrix $B(C)$ such that Eq. (1) holds if and only if Eq. (3) holds, but the number of rows of $B$ may not be minimal with this property. Then, in a second step, one eliminates redundant rows from $B$. We need the following definition about manipulations $M^{/ k}$ and $M_{\sim k}$ of a matrix $M$.

Definition. Let $M \in R^{m-\times d}$ and $k \leq d$.

(i) Let $M^{/ k} \in R^{\bar{m} \times d}$ (where typically $\overline{\mathrm{m}}>m$ ) be the matrix whose rows are given (in some arbitrary sequence) by

- the rows $c_{i}$ of $M$, for all $i$ with $c_{i k}=0$ and

- the rows $c_{i k} c_{j}-c_{j k} c_{i}$ for all $i, j$ with $c_{i k}>0$ and $c_{j k}<0$, where by $c_{i k}$ we denote the element of $M$ in row $i$ and column $k^{6}$

(ii) Let $M_{\sim k}$ be the matrix that results from $M$ by eliminating its $k$-th column.

(iii) Let $P(M) \equiv\left\{x \in R^{d}: M x \leq 0\right\}$.

(iv) For a set $S \subset R^{d}$ define the projection of $S$ in the direction of the $k$-th-unit vector $e_{k}, \operatorname{proj}_{k}(S) \equiv\left\{x \in R^{d}: x_{k}=0\right.$, $\left.\exists y \in R: x+y e_{k} \in S\right\}$, where $x_{k}$ denotes the k-th-component of $x$.

\footnotetext{
${ }^{6}$ Note that an upper bound for $\bar{m}$ is given by $m^{2} / 4$. The number $\bar{m}$ depends on the number of nonzero components in the $k$-th-column of $C$. The matrix $C^{/ k}$ is defined up to the ordering of its rows.
}

Note that it is trivial to calculate $M^{\prime k}$ and $M_{\sim k}$ for a given matrix $M$.

Algorithm 1. Set $H_{0}=C$ and $m_{0}=m$. Step 1: Define $H_{i+1}$ $\in R^{m_{i}+1 \times(d-1-i)}$ and $-B_{i+1} \in R^{m_{i}+1 \times m_{i}}$ as the first $d-1-i$ and last $m_{i}$ columns of the $d-1-i+m_{i}$ column matrix $\left(\left[H_{i}-I_{m_{i}}\right]^{11}\right)_{\sim 1}$, respectively. Iterate over Step 1 for $i=0, \ldots, d-1$. Then, set $B \equiv$ $-B_{d} \times \ldots \times B_{1}$.

Theorem 2 shows that the matrix $B=B(C)$ thus obtained is such that Eq. (1) has a solution in $\theta$ if and only if $B \mu \leq 0$. Using Farkas' Lemma we can then eliminate rows in $B$ that are redundant and obtain the desired recharacterization.

The justification of Algorithm 1 is based on Theorem 2 that provides a method of iteratively reducing the dimension of the parameter $\theta$ by one in each step. Iterating $d$ times eliminates $\theta$. More precisely, applying the theorem once, tells us that Eq. (1) has a solution in $\theta$ if and only if $\exists \bar{\theta} \in R^{d-1}$ such that $H_{1} \bar{\theta} \leq B_{1} \mu$. In the next step, apply the theorem with $H_{1}$ and $B_{1} \mu$ playing the role of $C$ and $\mu$, respectively. Iterating $d$ times, we obtain the $B$ in Algorithm 1.

Theorem 2. 1. $\exists \theta \in R^{d}$ s.t. $\left[C-I_{m}\right]\left[\begin{array}{l}\theta \\ \mu\end{array} \leq 0\right.$ if and only if 2. $\exists \bar{\theta} \in R^{d-1}$ s.t. $\left(\left[C-I_{m}\right]^{/ 1}\right)_{\sim 1}\left[\begin{array}{c}\bar{\theta} \\ \mu\end{array}\right] \leq 0$.

Proof. Note that the next two statements are equivalent to statement 1 in Theorem 2.

3. $P\left(\left[C-I_{m}\right]\right)$ contains a point $\left[\begin{array}{l}\theta \\ v\end{array}\right] \in R^{d+m}$ with $v=\mu$

4. $\operatorname{proj}_{1}\left(P\left(\left[C-I_{m}\right]\right)\right)$ contains a $\left[\begin{array}{l}\theta \\ v\end{array}\right] \in R^{d+m}$ with $v=\mu$ (and of course $\left.\theta_{1}=0\right)$.

The challenge now is to reduce the dimension of $\theta$. To do this, we use the following version of the Fourier-Motzkin elimination, see Ziegler (1994, Theorem 1.4) for a proof.

Lemma 1. Let $C \in R^{m \times d}$ and $k \leq d$. Then $\operatorname{proj}_{k}(P(C))=P\left(C^{k}\right)$ $\cap\left\{x \in R^{d}: x_{k}=0\right\}$.

Using Lemma 1, we find that the following statements are equivalent to statement 4 :

5. $\left.P\left(\left[C-I_{m}\right]\right]^{\prime 1}\right) \cap\left\{x \in R d+m: x_{1}=0\right\}$ contains a point $\left[\begin{array}{l}\theta \\ v\end{array}\right]$ with $v=\mu$ and $\theta_{1}=0$.

6. $\left.\left\{\begin{array}{l}\theta \\ v \\ v\end{array}\right] \in R^{d+m}:\left[C-I_{m}\right]^{/ 1}\left[\begin{array}{l}\theta \\ v\end{array}\right] \leq 0, \theta_{1}=0\right\}$ contains a $\left[\begin{array}{l}\theta \\ v\end{array}\right]$ with
$v=\mu, \theta_{1}=0$.

But 6 is clearly equivalent to the second statement in the theorem.

\section{References}

Andrews, D.W.K., Guggenberger, P., 2005. Validity of Subsampling and "Plug-in Asymptotic" Inference for Parameters Defined by Moment Inequalities, Cowles Foundation Discussion paper 1620.

Andrews, D.W.K., Berry, S., Jia, P., 2004. Confidence Regions for Parameters in Discrete Games with Multiple Equilibria, with an Application to Discount Chain Store Location, working paper.

Beresteanu, A., Molinari, F., 2005. Asymptotic Properties for a Class of Partially Identified Models, working paper.

Chernozhukov, V., Hong, H., Tamer, E., 2007. Estimation and confidence regions for parameter sets in econometric models. Econometrica 75, 1243-1284.

Gourieroux, C., Holly, A., Monfort, A., 1982. Likelihood ratio test, Wald test, and Kuhn-Tucker test in linear models with inequality constraints on the regression parameters. Econometrica 50, 63-80. 
Imbens, G.W., Manski, C.F., 2004. Confidence intervals for partially identified parameters. Econometrica 72, 1845-1857.

Kudo, A., 1963. A multivariate analog of the one sided test. Biometrika 50, 403-418.

Manski, C., 2003. Partial Identification of Probability Distributions. Springer, New York.

Manski, C.F., Tamer, E., 2002. Inference on regressions with interval data on a regressor or outcome. Econometrica 70, 519-546.

Moon, H.R., Schorfheide, F., 2004. Boosting your instruments: estimation with overidentifying inequality moment conditions. Working paper.

Pakes, A., Porter, J., Ho, K., Ishii, J., 2005. Applications of moment inequalities. Unpublished working paper, Department of Economics, Harvard University.
Romano, J.P., Shaikh, A.M., 2006. Inference for Identifiable Parameters in Partially Identified Econometric Models, working paper.

Rosen, A.R., 2005. Confidence Sets for Partially Identified Parameters that Satisfy a Finite Number of Moment Inequalities, working paper.

Savin, N.E., 1984. Multiple hypothesis testing. In: Griliches, Z., Intriligator, M.D. (Eds.), Handbook of Econometrics, vol. II. North-Holland, Amsterdam.

Wolak, F.A., 1991. The local nature of hypothesis testing involving inequality constraints in nonlinear models. Econometrica 59, 981-995.

Ziegler, G.M., 1994. Lectures on Polytopes. Springer, New York. 